\title{
Exciton transport in organic semiconductors: Förster resonance energy transfer compared with a simple random walk
}

\author{
K. Feron, ${ }^{1,2, a)}$ X. Zhou, ${ }^{1}$ W. J. Belcher ${ }^{1}$, and P. C. Dastoor ${ }^{1}$ \\ ${ }^{1}$ Centre for Organic Electronics, University of Newcastle, Callaghan, NSW 2308, Australia \\ ${ }^{2}$ CSIRO Energy Technology, Newcastle, NSW 2300, Australia
}

(Received 18 October 2011; accepted 14 January 2012; published online 29 February 2012)

\begin{abstract}
Förster resonance energy transfer theory (FRET) and a simple random walk (RW) are both implemented in a dynamic Monte Carlo simulation with the aim of determining the exciton diffusion length from photoluminescence (PL) measurements. The calculated diffusion lengths obtained from both models are shown to be the same. As such, given that the computational time of a random walk is typically 2-3 orders of magnitude smaller than the FRET approach, this work shows that the RW methodology can be a preferable model for the determination of diffusion lengths. We also show that the RW approach may also be implemented in Monte Carlo simulations that describe organic solar cells. Despite the fact that (compared with FRET) RW does not account for non-nearest neighbor hopping or energy relaxation, we show that the resulting overestimation of the simulated current will not exceed $2 \%$ for typical OPV parameters. In addition, by taking advantage of the gain in speed we are able to investigate the impact of the exciton diffusion length on the optimal interface distance and show that materials with longer exciton diffusion lengths are less sensitive to variations in the morphology of the active layer of an organic solar cell. (C) 2012 American Institute of Physics. [doi:10.1063/1.3687373]
\end{abstract}

\section{INTRODUCTION}

The performance of organic photovoltaics (OPVs) is highly susceptible to morphology variations on the nanoscale and, consequently, there have been a number of studies modeling the structure-function relationship in these devices. ${ }^{1-4}$ Monte Carlo modeling is a common computational approach for investigating morphological effects on the properties of organic semiconductors at the nanoscale and their impact on device performance. ${ }^{5}$ In these models a lattice is created where each voxel represents an individual unit of either electron donating or electron accepting material. In the case of exciton diffusion modeling the voxel size is determined typically by the degree of delocalization of the exciton itself. The spatial extent of excitons has been determined to be in the order of $1 \mathrm{~nm}$ and thus a voxel size of $1 \times 1 \times 1 \mathrm{~nm}^{3}$ is common. ${ }^{6}$ The performance of an OPV device is a function of the efficiency of both exciton dissociation and charge extraction. ${ }^{1}$ The exciton dissociation efficiency depends upon the morphology and the exciton transport properties associated with the materials of interest. ${ }^{7}$ Exciton diffusion is usually modeled using Forster resonance energy transfer theory (FRET), ${ }^{1,3,4,8}$ although other theories such as Dexter theory have been used depending on the dominant type of exciton (triplet or singlet). ${ }^{9}$

In FRET, the exciton hopping rate decreases as $R_{i j}{ }^{6}$, where $R_{i j}$ is the distance between the two lattice sites, $i$ and $j$. As such, in order to model exciton transport with FRET, the Förster radius, $R_{0}$, has to be determined for the materials in question. There exists a cubic relationship between the exciton diffusion length, $\mathrm{L}$, and $R_{0}$, and once this relationship is

\footnotetext{
a) Author to whom correspondence should be addressed. Electronic mail: krishna.feron@csiro.au.
}

exactly known the two can be easily inter-converted. ${ }^{10}$ Athanasopoulos et al. ${ }^{11}$ have shown that energetic disorder in the material diminishes $\mathrm{L}$, which means that there is an interplay between $\mathrm{L}, R_{0}$, and energetic disorder. Once two of these variables are known the third one can be determined. In the context of Monte Carlo modeling of OPV devices it is desirable to eliminate $R_{O}$ as a fitting parameter and determine it in an independent measurement. Determining $R_{0}$ can be achieved using photoluminescence (PL) measurements, which have previously been used to determine $\mathrm{L}$ for a variety of materials. ${ }^{9,12-14} \mathrm{PL}$ is the simplest method to determine $\mathrm{L}$, because one only needs to consider excitons and charge transport intricacies ${ }^{5}$ can be ignored. In addition the interfaces (substrate-material, material-air) are well defined, allowing good agreement between the model and experiment to be achieved because perfect interfaces are assumed in Monte Carlo models.

The implementation of FRET in Monte Carlo simulations is a computationally time consuming approach, especially when modeling large systems, i.e., thick layers. Here we show that a computationally fast alternative, a simple random walk (RW) in three dimensions, can be used to fit PL measurements and results in exciton diffusion lengths that are indistinguishable (considering the noise inherent in the measurements) from those obtained using the FRET methodology. We also explore the option of using RW in Monte Carlo models that describe OPV devices. The impact that the diffusion length has on the exciton dissociation efficiency is also discussed.

\section{SIMULATION METHODOLOGY}

In the simulations presented, the photoluminescent material is modeled as a cubic lattice of $100 \times 100 \times d$ sites 


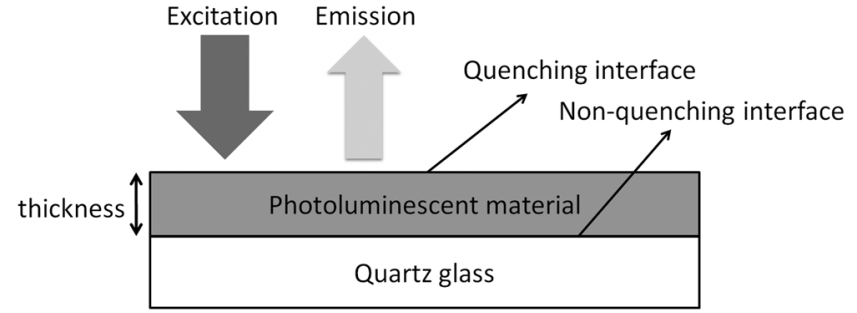

FIG. 1. Schematic of a PL measurement.

sitting on a quartz substrate, where $d$ is the thickness of the photoluminescent film (see Fig. 1). The voxel size is set at $1 \times 1 \times 1 \mathrm{~nm}$. Excitons are then created within this model proportionate to the optical field in the layer.

Two methods were used to simulate exciton transport in this model system. The first one uses FRET, where the hopping time, $t_{F R E T}$, between any two sites $i$ and $j$, separated by a distance $R_{i j}$, is given by,

$$
t_{F R E T}=t_{0}\left(\frac{R_{i j}}{R_{0}}\right)^{6} \frac{1}{f\left(E_{i}, E_{j}\right)}
$$

Here, $t_{0}$ is the exciton lifetime, $R_{0}$ the Förster radius, and $f\left(E_{i}, E_{j}\right)$ is a function accounting for energetic disorder such that:

$$
f\left(E_{i}, E_{j}\right)=\left\{\begin{array}{cc}
e^{-\frac{E_{j}-E_{i}}{k_{B} T}}, & E_{j}>E_{i} \\
1, & E_{j}<E_{i}
\end{array}\right.
$$

The energies of all sites are randomly assigned according to a Gaussian distribution with a standard deviation, $\sigma$, extracted from Gaussians fitted to the absorption spectrum as described in Scheidler et al. ${ }^{8}$ All hopping rates within a $3 \mathrm{~nm}$ radius (i.e., 122 sites) are considered and the site with the highest hopping probability is chosen to be the next destination of the exciton. The second method uses a simple random walk in 3 dimensions. There the average number of hops that an exciton takes in its lifetime, $N$, is given by,

$$
N=L^{2}\left\langle a^{2}\right\rangle
$$

and

$$
N=\frac{t_{0}}{t_{R W}}
$$

The average hopping time is thus given by,

$$
t_{R W}=\frac{t_{0}}{L^{2}\left\langle a^{2}\right\rangle},
$$

where $L$ is the diffusion length; $a$ is the lattice constant (1 $\mathrm{nm})$, and $t_{R W}$ is the hopping time. These times are queued and events executed according to Gillespie's algorithm. ${ }^{15}$ For both models, processes are assumed to decay exponentially with time, such that:

$$
t=-\log (X) t_{\text {event }},
$$

where $X$ is a random number between 0 and 1 and $t_{\text {event }}$ is the time of the event (i.e., the hopping time or the lifetime). The interface of the photoluminescent layer with the quartz substrate is treated as an inert interface, ${ }^{12}$ while the air interface is treated as a quenching interface, because molecular oxygen is known to be an efficient collisional quencher. ${ }^{16} \mathrm{~A}$ quenching probability is assigned when an exciton reaches this interface. If an exciton is successfully quenched, it is deleted from the system. If an exciton is not quenched within its lifetime, it will recombine and emit a photon. Bulk hetero-junction morphologies were created using a cellular automata method. ${ }^{17}$ First each lattice site is assigned a random moiety identifier ( 0 for the electron accepting moiety and 1 for the electron donating moiety), while maintaining a 1:1 volume ratio. Attempts are made to swap two neighboring pairs of sites. Swaps are accepted with a probability of,

$$
P(\Delta E)=\frac{\exp \left(-\frac{\Delta E}{k_{B} T}\right)}{1+\exp \left(-\frac{\Delta E}{k_{B} T}\right)},
$$

where $\Delta \mathrm{E}$ is the energy difference between the state after the swap and that before the swap. The energy of a site is calculated using,

$$
E_{i}=-\frac{J}{2} \sum_{j} \delta_{s_{i}, s_{j}}-1,
$$

where $J$ is set to $k_{B} \mathrm{~T}$ and $s$ is the moiety identifier. The summation over $j$ indicates a summation overall nearest neighbors. Periodic boundaries are used in all six directions in order to prevent edge effects. As sites are swapped, the feature size (ratio of volume and interface area) of the blend increases. The swapping process continued until a feature size of approximately $4.5 \mathrm{~nm}$ was reached. These morphologies were used to explore the applicability of RW in OPV models. As opposed to the PL experiment, OPV devices have two active components and excitons are dissociated with a set dissociation probability at the interface between the two moieties, giving rise to a charge pair. To investigate the effect of using RW instead of FRET on subsequent processes in an OPV device, charge transport was also simulated using a first reaction method. ${ }^{18}$ The hopping behavior of the created charges is simulated using Marcus theory:

$$
t_{\text {hop }}=\frac{1}{w_{0}} \exp \left(\frac{\left(E_{j}-E_{i}+\lambda\right)^{2}}{4 \lambda k_{B} T}\right),
$$

Here $\lambda$ is the reorganization energy (chosen to be $0.5 \mathrm{eV}$ ), $w_{0}$ is a prefactor that is set to $10^{9} \mathrm{~s}^{-1}$, and $\mathrm{E}_{\mathrm{i}}$ and $\mathrm{E}_{\mathrm{j}}$ are the energies of two adjacent sites in consideration that include Coulombic interaction, electric field, and energetic disorder. The electric field was set to $10^{7} \mathrm{~V} / \mathrm{m}$, which approximately corresponds to the field in an OPV device under short-circuit conditions. Coulombic interaction is calculated for charges within the thermal capture radius $+1 \mathrm{~nm}$. The thermal capture radius, $r$, is defined as:

$$
r=\frac{q^{2}}{4 \pi \varepsilon_{0} \varepsilon_{r} k_{B} T},
$$

where $q$ is the charge of an electron, $\varepsilon_{0}$ the permittivity of a vacuum, and $\varepsilon_{\mathrm{r}}$ the dielectric constant, which was chosen to 
be 4. For this value of $\varepsilon_{r}$, the Coulomb interaction is calculated for charges within a radius of $15 \mathrm{~nm}$. Adjacent charge pairs can geminately recombine with a recombination rate of $10^{7} \mathrm{~s}^{-1}$. Charge pairs are considered fully separated when the distance between the two respective charges is larger than the thermal capture radius $+1 \mathrm{~nm}$. The lattice size used for the charge transport simulations was $50 \times 50 \times 50 \mathrm{~nm}$, but periodic boundary conditions were used in each direction, resulting in an effectively infinite volume.

Exciton hopping simulations were run on personal computers equipped with Intel Pentium Dual Core $2.0 \mathrm{GHz}$ processors. Full OPV device simulations were run using the CSIRO Supermicro Xeon GPU Cluster.

\section{RESULTS AND DISCUSSION}

A validation of the Monte-Carlo simulation is provided by Fig. 2, which shows the variation in calculated exciton diffusion length as a function of Förster radius for three values of energetic disorder, $\sigma$, corresponding to $0.09 \mathrm{eV}, 0.05 \mathrm{eV}$, and a perfect energy landscape (no energetic disorder). As energetic disorder increases, the exciton diffusion length decreases and, importantly, in all three cases the expected cubic relationship is observed between the Förster radius and diffusion length. ${ }^{10}$ Small molecules generally exhibit larger $\mathrm{L}$ than polymers,${ }^{14}$ which is a result of small energetic disorder. ${ }^{11}$

In order to compare the two models used in the Monte Carlo simulations we define the effective distance traveled by an exciton as the distance between the point of exciton creation and exciton recombination. Figure 3 shows the distribution of these effective distances obtained using the FRET and RW models and demonstrates that both models give almost identical distributions.

In FRET the hopping probability decreases rapidly with distance and thus hopping to nearest neighbors is dominant. Therefore, if hops to sites further away than one lattice constant occur rarely, implementing FRET simply becomes a computationally more time consuming way of performing a random walk in three dimensions. For $\sigma$ values typically found for organic materials $(10-90 \mathrm{meV})^{4,8,19,20}$ the most frequent hop will be a nearest neighbor hop. Only for $\sigma$ values significantly larger than $k_{B}$ T will the energy landscape dominate the diffusion behavior in such a way that the modeled exciton transport will no longer behave like a RW. In

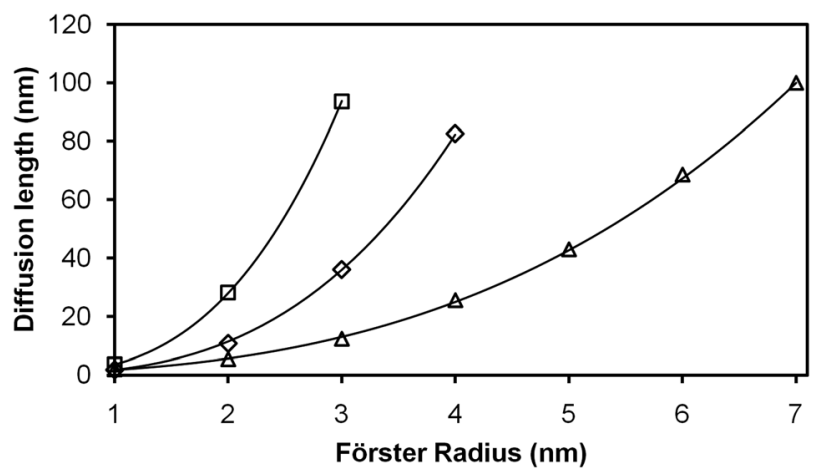

FIG. 2. Cubic relationship between $\mathrm{L}$ and $R_{0}$ for $\sigma=0.09 \mathrm{eV}$ (squares), 0.05 $\mathrm{eV}$ (diamonds), and $0 \mathrm{eV}$ (triangles). The solid lines are cubic fits used to intrapolate between points.

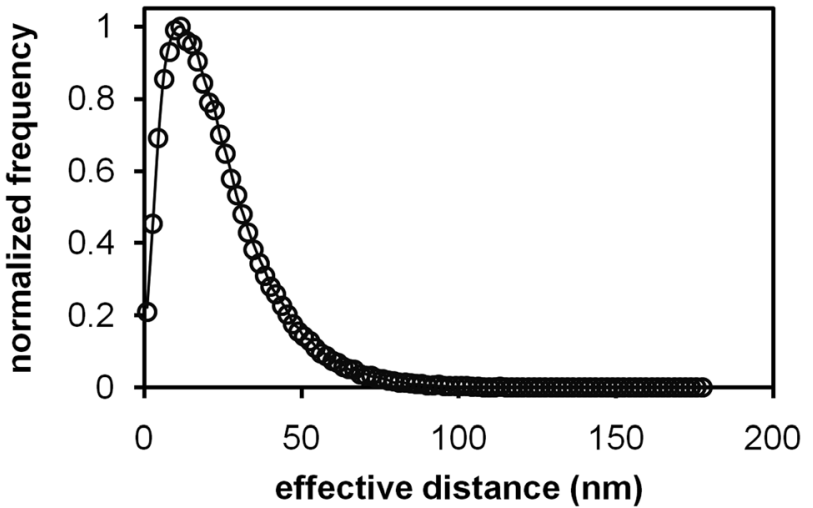

FIG. 3. The distance between the point of exciton creation and exciton recombination is the effective distance traveled. The distribution of the effective distances traveled by excitons for FRET and RW are indicated with open circles and a solid line, respectively. The FRET simulation was executed with $R_{0}=2.75 \mathrm{~nm}$ and $\sigma=0.05 \mathrm{eV}$. These parameters give a corresponding value of $\mathrm{L}=28 \mathrm{~nm}$, which was used for the RW simulation. The lifetimes were the same in both simulations $\left(1 \times 10^{-6} \mathrm{~s}\right)$.

this case, the hopping behavior will closely follow low energy pathways in the energy landscape.

Other approaches used to describe triplet excitons, such as Dexter theory ${ }^{9,21}$ and Miller-Abrahams tunneling, ${ }^{22}$ also involve the possibility of hops to non-adjacent lattice sites. However, in both of these cases, the probability of hops to sites that are more than one lattice site away decreases exponentially with distance. Therefore, it seems reasonable to suggest that the RW model will be equivalent to these approaches as well.

Given that both the FRET and RW models display the same effective distance distribution, we might expect that calculating the PL as a function of layer thickness for these two models should also produce the same result. However, two possible sources of discrepancy between the two models are the effect of exciton generation rate and interface quenching probability on the calculated PL.

It is possible for the exciton diffusion behavior to change if the exciton density is extremely large, because many sites will be occupied and thus the number of available sites for each hop will be diminished. In order to test the sensitivity of the simulation to this parameter, the exciton generation rate was varied up to 10 excitons $/ \mathrm{s} / \mathrm{nm}^{3}$ for the RW model, which is well beyond the light intensity used in obtaining the experimental data in Fig. 5. However, no change in the exciton diffusion behavior with exciton generation rate was observed. As such, the PL signal is independent of exciton generation rate $\left(<10\right.$ excitons $\left./ \mathrm{s} / \mathrm{nm}^{3}\right)$ and thus we can normalize the PL signal relative to that of the thickest modeled system.

Figure 4 shows the effect of the quenching probability on the normalized PL signal as a function of thickness for the RW model. It is apparent from Fig. 4 that there is hardly any statistical difference between the normalized PL results observed for a quenching probability of 50\% and that for $100 \%$. Indeed, reducing the quenching probability to only $1 \%$ still results in only a small change in normalized PL signal. The reason for this observation is that once an exciton is in the vicinity of the quenching interface it is likely that it 


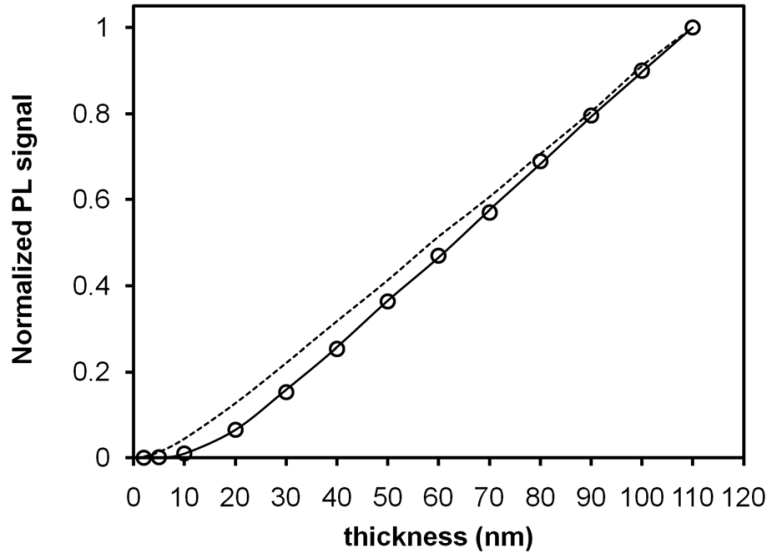

FIG. 4. PL as a function of thickness for quenching probabilities of $1 \%$ (dashed line), 50\% (open circles), and 100\% (solid line). L was set to $40 \mathrm{~nm}$ for all three simulations.

hits the interface several times, thus significantly increasing the chances of quenching. Moreover, given that molecular oxygen is known to be a very efficient quencher ${ }^{16}$ and that the simulation has a low sensitivity to quenching probability it seems reasonable to model the quenching probability at the air interface as $100 \%$.

To further demonstrate the validity of the RW model, we have compared calculated PL measurements as a function of thickness to experimental data from the literature.

Figure 5 shows a plot of normalized PL as a function of thickness for $6 \mathrm{~T}$ for both the simulation and experimental results obtained from Mani et al. ${ }^{12}$ In the absence of any information about the internal optical field extant during the experiment, a uniform optical field was assumed. However, regardless of the validity of this simplification, it provides a good case to compare FRET to RW in terms of L extracted from fitting and computational efficiency. Figure 5 can be separated into three sections. In section 1, quenching is the dominant process, because most excitons are in the vicinity of the air interface and very little PL is observed. The linear regime in section 3 arises from the fact that increasing the thickness in this regime only results in greater recombination

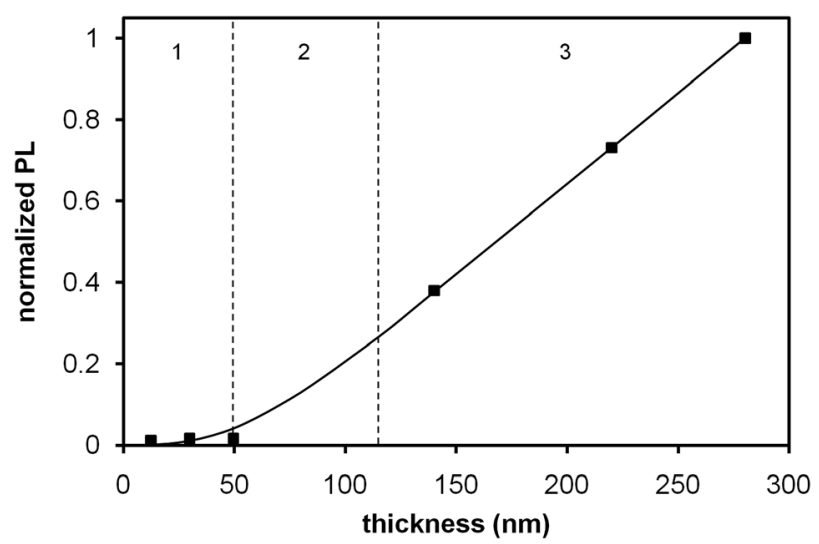

FIG. 5. Normalized PL signal as a function of $6 \mathrm{~T}$ layer thickness. The solid line is obtained from the simulation using the RW model for a fitted diffusion length of $140 \mathrm{~nm}$. The experimental results (filled squares) are obtained from Mani et al. ${ }^{12}$ Two vertical dashed lines have been added to distinguish between the three characteristic regimes associated with the measurement.
TABLE I. Comparison of computational time cost for the FRET and RW models.

\begin{tabular}{lcc}
\hline \hline Model & $\begin{array}{c}\text { Calculated Exciton } \\
\text { Diffusion Length (nm) }\end{array}$ & $\begin{array}{c}\text { Computational } \\
\text { time (s) }\end{array}$ \\
\hline FRET & 140 & $2.0 \times 10^{6}$ \\
RW & 140 & $6.5 \times 10^{3}$ \\
\hline \hline
\end{tabular}

while the number of excitons that are quenched does not change, while section 2 is a transitional phase between sections 1 and 3. It is clear that the modeled fit is in close agreement with the experimental data across the different sections, thus providing a further confirmation of the validity of the RW approach.

The key advantage of the RW model from a computational point of view is that it is computationally 2-3 orders of magnitude faster than other approaches, such as FRET. As such, the RW approach is particularly suited to modeling large lattices, i.e., thick films. The significant saving in computational time that can be gained by using RW is illustrated in Table I, which compares the time taken to simulate the experimental data shown in Fig. 5 for both the RW and FRET models. Both models give the same calculated threedimensional exciton diffusion length of $140 \mathrm{~nm}$, which is equivalent to a one-dimensional exciton diffusion length of $81 \mathrm{~nm}$ and corresponds well with a previously reported value in the literature of $100 \pm 20 \mathrm{~nm}^{23}$ However, the FRET model takes more than 23 days of computational time whereas the RW model is complete in under $2 \mathrm{~h}$, more than 300 times faster. As such, RW is the preferred model for determining $\mathrm{L}$ from PL measurements.

Now we explore the possibility of implementing RW in Monte Carlo models that describe OPV devices. There are two key differences between the RW and FRET methodologies. First, RW only considers nearest neighbors whereas in systems with small material domain sizes and low quenching probability it is necessary to consider hops to sites that are further away than one lattice constant. Figure 6 shows exciton dissociation efficiency as a function of $\mathrm{L}$ for a bulk

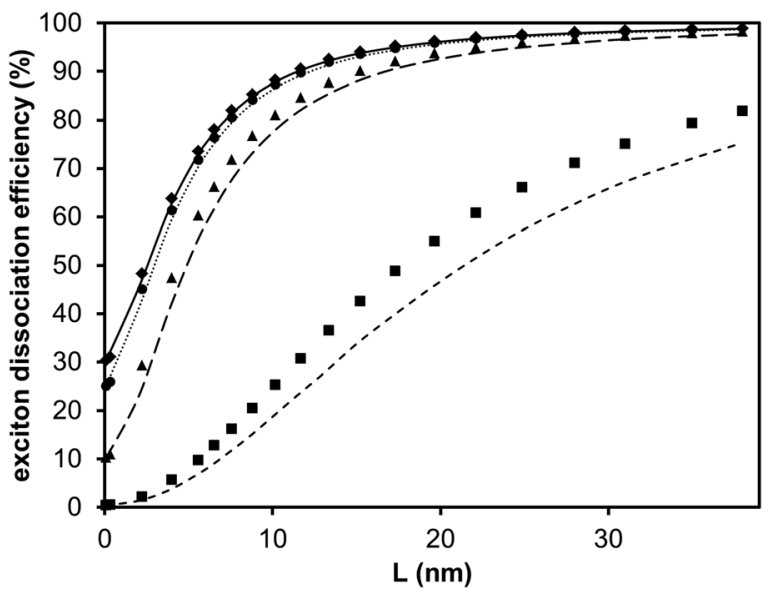

FIG. 6. Exciton dissociation effiency as a function of L for FRET (lines) and RW (markers). Graphs for the following dissociation probabilities are shown: $100 \%$ (diamonds and solid line), $75 \%$ (circles and dotted line), $25 \%$ (triangles and long-dashed line), and 1\% (squares and short-dashed line). 


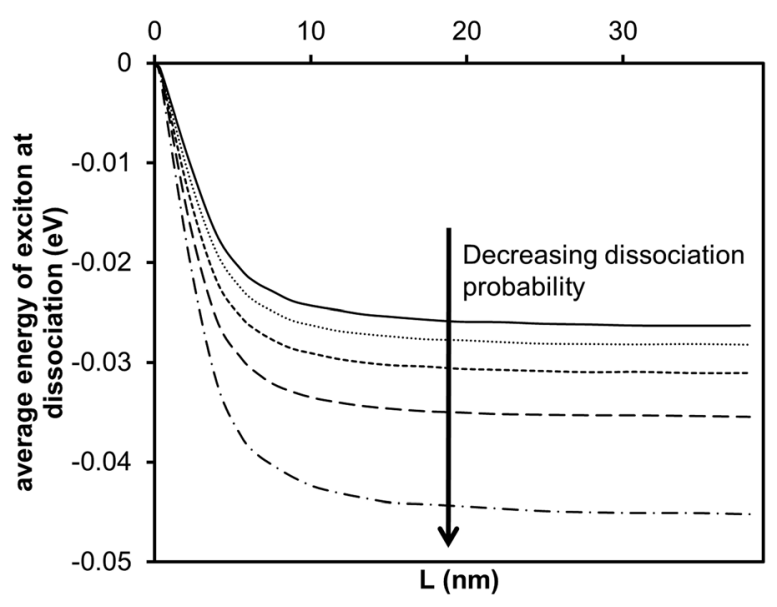

FIG. 7. Average energy of an exciton at dissociation as a function of $\mathrm{L}$ for a BHJ morphology with a feature size of $4.5 \mathrm{~nm}$ when using FRET. $\sigma$ was chosen to be $0.05 \mathrm{eV}$. Graphs shown are for the following dissociation probabilities: $100 \%$ (solid line), $75 \%$ (dotted line), 50\% (short-dashed line), $25 \%$ (long-dashed line), and 1\% (dash-dot line).

heterojunction $(\mathrm{BHJ})$ morphology with a feature size of 4.5 $\mathrm{nm}$. While it is clear that lowering the dissociation probability will decrease the exciton dissociation efficiency, the drop-off is different for RW compared with FRET.

For less efficient hetero-interfaces, RW will only match FRET if the diffusion length is sufficiently large compared with the feature size of the morphology. As mentioned before, large diffusion lengths are observed for small molecules. $^{11,14}$ In terms of the exciton dissociation efficiency, there is no difference between FRET and RW if the dissociation probability is high $(>75 \%)$.

The second limitation of RW is that it does not consider energy relaxation of the exciton as it hops from site to site. Figure 7 shows that moderate energy relaxation occurs for systems with high exciton dissociation probabilities and substantial energy relaxation occurs for systems with low exciton dissociation probabilities.

(A)

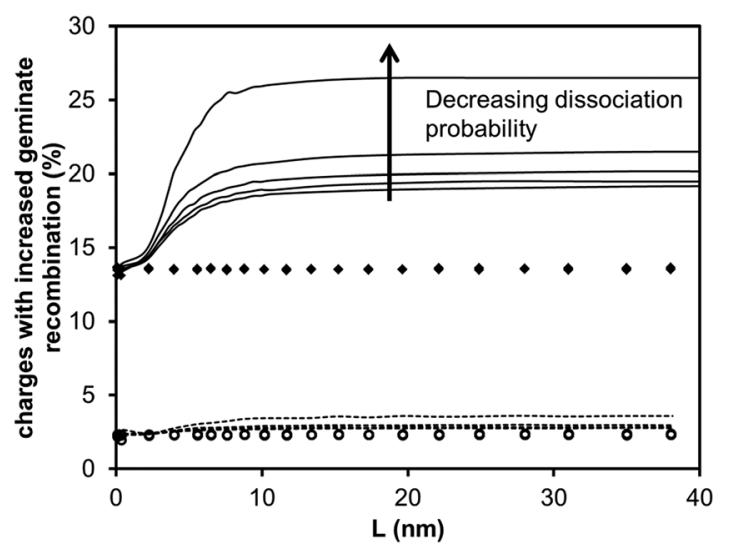

In Monte Carlo models exciton dissociation occurs at hetero-interfaces with a set dissociation probability, the energy of the exciton is not taken into account in this process. While exciton relaxation has little to no impact on the exciton diffusion process itself or even the exciton dissociation process, it will cause excitons to preferentially dissociate at interface sites with low energies. In other words, newly dissociated charges will have lower energies when using FRET instead of RW. Groves et $a .^{24}$ have shown recently that trapped charges (charges with a significantly lower energy compared with that of its surroundings) will have an increased probability of geminate recombination. The effect of trapping is not straightforward, because energy relaxation will cause more electrons to be trapped, but the opposite is true for holes. Groves et al. demonstrated that, for a bilayer device, a decrease in geminate pair separation efficiency is observed when both electron and hole are trapped with energy of more than $0.05 \mathrm{eV}$ or when either charge carrier is trapped with energy of more than $0.15 \mathrm{eV}^{24}$ In order to determine the significance of this charge trapping effect, the percentage of newly created charges that satisfy these conditions has been determined for both FRET and RW (Fig. 8(a)).

In the case of RW, there is no dependency of geminate recombination on $\mathrm{L}$ or the exciton dissociation probability (the graphs exactly overlay) because charges are randomly created at interfaces. Here, only the roughness of the energy landscape will influence geminate recombination. Thus, for very small L values, FRET will give the same result as RW, because excitons will hardly hop between sites and thus energy relaxation cannot occur. Exciton dissociation is then mainly determined by the location of exciton creation, which is a random process for $\mathrm{BHJ}$ morphologies. As L increases, the probability of geminate recombination will increase until a maximum is reached when $\mathrm{L}$ equals roughly twice the feature size $(\sim 10 \mathrm{~nm})$. For $\sigma=0.05 \mathrm{eV}$, there is very little difference between RW and FRET especially for high

(B)

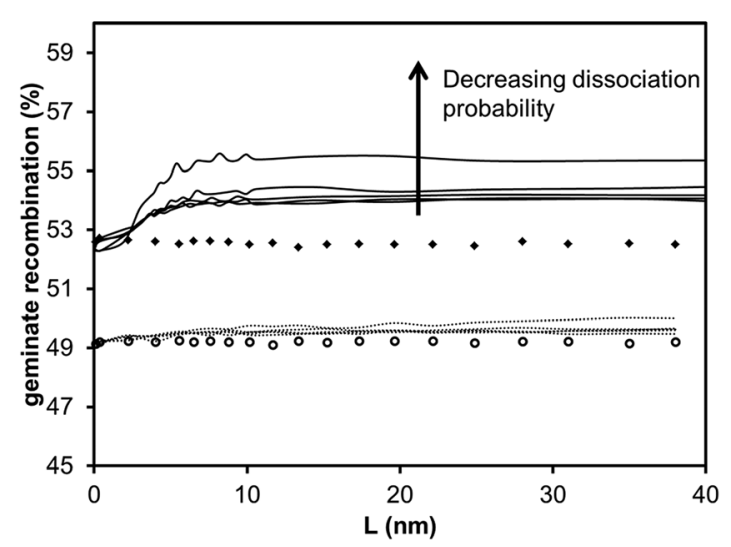

FIG. 8. (a) Percentage of charges meeting the conditions of reduced geminate separation efficiency (i.e., when both hole and electron are trapped with energy $>0.05 \mathrm{eV}$ or either charge is trapped with energy $>0.15 \mathrm{eV}$ ), ${ }^{24}$ as a function of L for RW (circles and diamonds) and FRET (solid and dashed lines). (b) Geminate recombination as a function of L for RW (circles and diamonds) and FRET (solid and dashed lines). In both (a) and (b) the dissociation probability was varied $(100 \%, 75 \%, 50 \%, 25 \%$, and $1 \%)$ for both RW and FRET. In both (a) and (b) the solid lines and diamonds correspond to $\sigma=0.1 \mathrm{eV}$ whereas the dashed lines and circles correspond to $\sigma=0.05 \mathrm{eV}$. The bottom plot of each series corresponds to a dissociation probability of $100 \%$ and the top plot corresponds to $1 \%$. There are 20 plots in each graph; however, the 10 plots for RW condenses to 2 plots, because the dissociation probability and diffusion length do not influence the geminate separation efficiency. 
dissociation probabilities. For material systems with high exciton dissociation probabilities $(>50 \%)$, FRET predicts that $2.8 \%$ of dissociated charges will have an increased geminate recombination probability due to trapping effects compared with $2.35 \%$ for RW. This is an insignificant difference and RW is readily applicable in this situation. However, larger differences are noticeable for materials with large energetic disorder. For $\sigma=0.1 \mathrm{eV}$, increased geminate recombination is found for $19-20 \%$ of all charges when using FRET compared with $13.6 \%$ for RW. It is noteworthy that geminate recombination is mainly caused by both charges being trapped as opposed to single trapped charges. For $\sigma=0.05 \mathrm{eV}$, only $0.1 \%$ of all charges have a trap energy of $0.15 \mathrm{eV}$ or higher. Even for large energetic disorder (e.g., $0.1 \mathrm{eV}$ ) this value is only $4.4 \%$ because energy relaxation decreases the occurrence of trapped holes, which partially compensates for the increased number of trapped electrons. Given that charges that meet the conditions of increased geminate recombination do not necessarily recombine, the data in Fig. 8(a) therefore provide an overestimation to recombination. To determine the exact effect of exciton energy relaxation on geminate recombination, charge transport has been modeled; see Fig. 8(b). To isolate the effect of geminate recombination on bulk geminate recombination, periodic boundaries were invoked in all six directions, which effectively means that image charge effects are not included. This approach is similar to that of Groves et al. ${ }^{24}$ as they modeled a bilayer where the hetero-interface is too far away from the electrodes for image charges to have a significant influence. Image charge effects are expected to increase geminate separation and, thus, the choice of not looking at edge effects near the electrode provides an upper limit. The graphs in Fig. 8(a) and 8(b) are similar in shape, which indicates that our simulations give similar results to those of
Groves et al. In both cases, geminate recombination increases as the dissociation probability decreases due to a decrease in the average exciton energy at dissociation (Fig. 7). The difference between RW and FRET is smaller in Fig. 8(b) compared with Fig. 8(a). This is consistent with the fact that the data in Fig. 8(a) are not geminate recombination, but the percentage of charges with an increased probability of geminate recombination. The maximum underestimation of geminate recombination when using RW is $3 \%$ and only $1.5 \%$ if the dissociation probability is $100 \%$.

Therefore, the overall effect of using RW instead of FRET on the simulated current is minimal. If we consider the worst case that we investigated (dissociation probability of $1 \%$ and $\mathrm{L}$ is such that the difference in exciton dissociation efficiency (Fig. 6) is maximized (10\%)), the simulated current will be overestimated by $13 \%$. However, these extreme values of $\sigma$ and dissociation probability are actually outside the range of what is normally accepted. The dissociation probability is generally taken as $100 \%$ and $\sigma$ generally ranges from 0.01 to $0.09 \mathrm{eV} .^{1,4,8,19,25}$ For values within the usual range, the overestimation will not be greater than $2 \%$ and negligible in most cases. This deviation is an acceptable error considering the typical variability in the performance of OPV devices. It has to be noted that the gain in computational efficiency is less significant in Monte Carlo models that simulate OPV devices, because charge transport is the bottleneck in these simulations.

It is widely recognized that domain size is a key factor in determining the performance of OPV devices. ${ }^{26}$ Monte Carlo simulation of exciton diffusion is one of the few techniques available for determining a priori the optimum domain size. Figure 9(a) shows the distribution of distances that excitons travel, i.e., the distance between the location of exciton generation and the location of recombination,
(A)

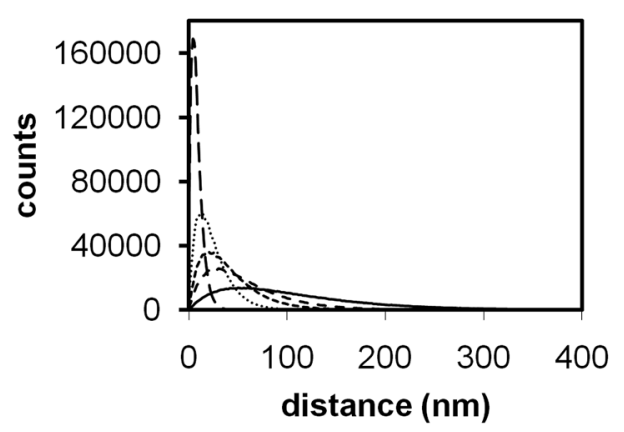

(B)

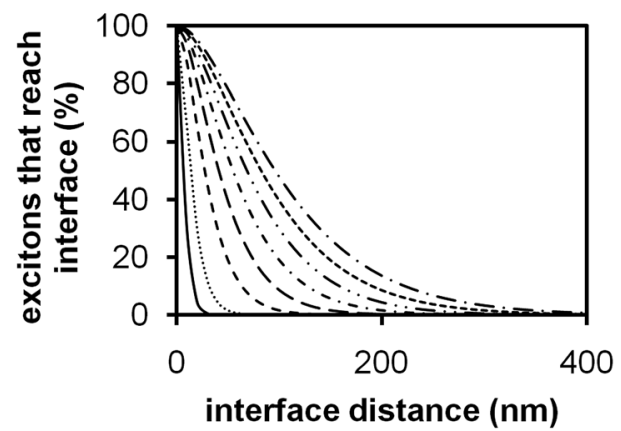

FIG. 9. (a) plots of effective distance traveled for a range of diffusion lengths. Traces are shown for $\mathrm{L}=10 \mathrm{~nm}, 30 \mathrm{~nm}$, $50 \mathrm{~nm}, 70 \mathrm{~nm}$, and $130 \mathrm{~nm}$. Smaller L exhibit narrower and higher peaks. (b) The percentage of excitons collected at an interface as a function of interface distance. Traces are shown for $\mathrm{L}=10 \mathrm{~nm}$, $20 \mathrm{~nm}, 40 \mathrm{~nm}, 60 \mathrm{~nm}, 80 \mathrm{~nm}, 100 \mathrm{~nm}$, $120 \mathrm{~nm}$, and $140 \mathrm{~nm}$. A shorter L exhibits a quicker drop off. The graph in (c) shows the derivative of (b) and this gives the same distribution as (a) with a sign change. Traces are shown for $\mathrm{L}=10 \mathrm{~nm}$, $30 \mathrm{~nm}, 50 \mathrm{~nm}, 70 \mathrm{~nm}, 90 \mathrm{~nm}, 110 \mathrm{~nm}$, and $130 \mathrm{~nm}$. A narrower and deeper minimum corresponds to a smaller L.

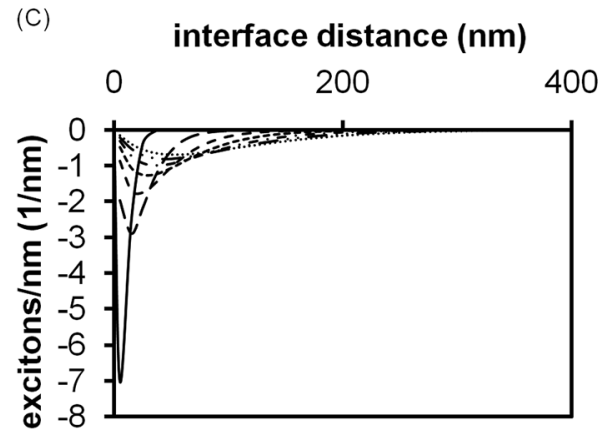


calculated for a range of exciton diffusion lengths. This distribution gives an indication of the optimal phase separation for a given diffusion length in the active layer of OPV devices. Figure 9(b) shows the minimum interface distance required to collect a certain fraction of excitons as a function of diffusion length. Here, the interface distance is defined as the absolute distance that an individual exciton has to travel to reach a hetero-interface and is analogous to the concept of domain size in a bulk heterojunction morphology.

Figure 9(b) suggests that only approximately $30 \%$ of all excitons are able to reach an interface that is one diffusion length away. This value is likely to be an upper limit because, for a given structure, not all excitons will travel directly to the closest interface. For example, in a columnar structure, two out of the six hopping directions will not lead to an interface.

The number of excitons that reach an interface drops off rapidly as the interface distance increases. The derivative of Fig. 9(b) has been calculated to determine at what distance most excitons are lost as the interface distance increases and is shown in Fig. 9(c). The minimum of the derivative is located at the same distance as the peak of the corresponding distribution of effective distances (Fig. 9(a)). It is therefore desirable for optimal exciton dissociation to design the morphology so that the average interface distance is smaller than the peak of the effective distance distribution. Figure 9(c) also shows that the minimum is deeper for shorter diffusion lengths and, as such, materials with shorter diffusion lengths are more susceptible to deviations from the optimum domain size. Conversely, the exciton dissociation efficiency of organic solar cells based on materials with longer diffusion lengths (i.e., less energetic disorder) will be more forgiving to variations in domain size. This work highlights the effectiveness of the RW approach in Monte Carlo simulations of exciton diffusion in OPV devices. The simulations presented in Fig. 9 required approximately one day of computational time using the RW approach. By contrast, the same simulations would have required almost half a year of computational time using the FRET model.

\section{CONCLUSION}

We have shown that a simple RW model gives the same hopping behavior as FRET when modeling single component systems. Thus the process of determining exciton diffusion lengths by fitting against PL measurements can be made less computationally expensive by using the RW model, because RW is typically $2-3$ orders of magnitude faster than FRET. This saving is especially significant for large systems, i.e., thick layers and materials with large exciton diffusion lengths such as $6 \mathrm{~T}$. The quenching efficiency has been shown to have little impact on the PL signal when efficient quenchers are used. Using the RW model, the exciton diffusion length of sexithiophene has been calculated to be $140 \mathrm{~nm}$, which is in good agreement with literature. Once L is known, $R_{O}$ can be determined using the cubic relationship between $\mathrm{L}$ and $R_{0}$. This means RW can be used to determine $R_{0}$, which is an essential parameter when using FRET. Despite the fact that RW does not account for non-nearest neighbor hopping or energy relaxation, the resulting overestimation of the simulated current does not exceed $2 \%$ for common OPV parameters. In addition, simulation results highlight that materials with large exciton diffusion lengths are less susceptible to structural variations that arise in fabrication processes.

${ }^{1}$ P. K. Watkins, A. B. Walker, and G. L. B. Verschoor, Nano Lett. 5, 1814 (2005).

${ }^{2}$ J. Ouyang, C.-W. Chu, F.-C. Chen, Q. Xu, and Y. Yang, J. Macromol. Sci. Part A Pure Appl. Chem. 41, 1497 (2004).

${ }^{3}$ L. Meng, Y. Shang, Q. Li, Y. Li, X. Zhan, Z. Shuai, R. G. E. Kimber, and

A. B. Walker, J. Phys. Chem. B 114, 36 (2010).

${ }^{4}$ F. Yang and S. R. Forrest, ACS Nano 2, 1022 (2008).

${ }^{5}$ M. Casalegno, G. Raos, and R. Po, J. Chem. Phys. 132, 094705 (2010).

${ }^{6}$ M. Knupfer, Synth. Met. 141, 21 (2004).

${ }^{7}$ Y. Shao and Y. Yang, Adv. Mater. 17, 2841 (2005).

${ }^{8}$ M. Scheidler, U. Lemmer, R. Kersting, S. Karg, W. Riess, B. Cleve, R. F. Mahrt, H. Kurz, H. Bässler, E. O. Göbel, and P. Thomas, Phys. Rev. B 54, $5536(1996)$

${ }^{9}$ R. R. Lunt, N. C. Giebink, A. A. Belak, J. B. Benziger, and S.R. Forrest, J. Appl. Phys. 105, 053711 (2009).

${ }^{10}$ K. A. Colby, J. J. Burdett, R. F. Frisbee, L. Zhu, R. J. Dillon, and C. J. Bardeen, J. Phys. Chem. A 114, 3471 (2010).

${ }^{11}$ S. Athanasopoulos, E. V. Emelianova, A. B. Walker, and D. Beljonne, Phys. Rev. B 80, 1 (2009).

${ }^{12}$ A. Mani, J. Schoonman, and A. Goossens, J. Phys. Chem. B 109, 4829 (2005).

${ }^{13}$ Y. C. Zhou, Y. Wu, L. L. Ma, J. Zhou, X. M. Ding, and X. Y. Hou, J. Appl. Phys. 100, 023712 (2006).

${ }^{14}$ P. Peumans, A. Yakimov, and S. R. Forrest, J. Appl. Phys. 93, 3693 (2003).

${ }^{15}$ D. Gillespie, J. Comput. Phys. 22, 403 (1976).

${ }^{16} \mathrm{~J}$. R. Lakowicz, Principles of Fluorescence Spectroscopy, 3rd ed. (Plenum Press, New York, 1983), p. 258.

${ }^{17}$ P. Peumans, S. Uchida, and S. R. Forrest, Nature 425, 158 (2003).

${ }^{18}$ J. Lukkien, J. Segers, P. Hilbers, R. Gelten, and A. Jansen, Phys. Rev. E $\mathbf{5 8 ,} 2598$ (1998).

${ }^{19}$ R. A. Marsh, C. Groves, and N. C. Greenham, J. Appl. Phys. 101, 083509 (2007).

${ }^{20} \mathrm{C}$. Madigan and V. Bulović, Phys. Rev. Lett. 96, 1 (2006).

${ }^{21}$ D. L. Dexter, J. Chem. Phys. 21, 836 (1953).

${ }^{22}$ A. Köhler and H. Bässler, J. Mater. Chem. 21, 4003 (2011).

${ }^{23}$ J. Kalinowski, Chem. Phys. 237, 233 (1998).

${ }^{24}$ C. Groves, J. C. Blakesley, and N. C. Greenham, Nano Lett. 10, 1063 (2010).

${ }^{25}$ C. Groves, R. A. Marsh, and N. C. Greenham, J. Chem. Phys. 129, 114903 (2008).

${ }^{26}$ X. Yang and J. Loos, Macromolecules 40, 1353 (2007). 